\title{
Der richtige Weg. Eine EU nach dem Vorbild Jean Monnets
}

\author{
Nikolaus Scholik
}

Online publiziert: 29. Oktober 2020

(C) Springer Fachmedien Wiesbaden GmbH, ein Teil von Springer Nature 2020

Zusammenfassung Der Beitrag befasst sich mit der derzeitigen Krise der Weiterentwicklung der Europäischen Union. Die andauernde Verzögerungstaktik kann und wird keine Lösung bringen. Ist die Union im Sinne Monnets noch zu retten? Oder andersherum gefragt: Welche Folgen hätte der Rückschritt auf eine reine Wirtschaftsgemeinschaft? Der Beitrag analysiert die Herausforderungen und Chancen eines alternativen Weges, der ganz im Sinne der richtigen Erkenntnis Monnets bereits vor vielen Jahrzehnten unerlässlich schien: mehr Bundesstaat als Staatenbund.

Schlüsselwörter Europäische Union · Europäischer Bundesstaat · Vereinigte Staaten von Europa · Jean Monnet · Internationale Herausforderungen ·

Außenpolitik

\section{The Right Way. An EU Modelled on Jean Monnet}

Abstract The article discusses the current crisis of the evolution of the European Union. The ongoing delaying tactics cannot and will not bring a solution. Is the Union still salvageable in Monnet's sense? Or to put it the other way around: what would be the consequences of regressing to a purely economic union? This article analyzes the challenges and opportunities of an alternative path that, in keeping with Monnet's correct insight, seemed indispensable many decades ago: more of a federal state than a confederation of states.

Keywords European Union · European federal state · United States of Europe · Jean Monnet · International challenges · Foreign policy

Mag. Dr. N. Scholik ( $\square)$

Wien, Österreich

E-Mail: ncscholik@aon.at 
Dear Father, we dream, we dream, we dream while we may. (Neil Diamond)

\section{Einleitung}

Seit nunmehr 71 beziehungsweise 69 Jahren $^{1}$ versuchen die europäischen Nationalstaaten aus den seit Jahrhunderten andauernden Kämpfen/Kriegen um Vormachtstellung, politische, wirtschaftliche, ideologische Positionen oder einfach Interessen und Machtanspruch auszubrechen und zu einer höheren, friedlichen Form des Zusammenlebens auf unserem Kontinent zu gelangen. Das Modell einer engeren Verflechtung - wirtschaftlich zunächst, politisch in der Folge, also zunächst eines Staatenbundes, der dann zu Vereinigten Staaten Europas mit föderaler Struktur führt, modellhaft den Vereinigten Staaten von Amerika entsprechend - schien dem Vater Europas, Jean Monnet, der richtige Weg. Er hat sich Zeit seines Lebens für dieses Ziel eingesetzt und in den Gründungsjahren mitgeholfen, Jahrhunderte alte Vorurteile und Steine aus den nationalen Wegen in Richtung dieses Ziels aus dem Weg zu räumen. Rasch musste er aber erkennen, dass nach dem noch einig vollzogenen ersten großen Schritt des wirtschaftlichen Zusammenschlusses, der so wichtige zweite nicht vorankommen wollte. Monnet starb im Frühjahr 1979, dem Jahr der ersten Direktwahl zum Europäischen Parlament und den ersten Schritten zur Einführung des Europäischen Währungssystems (EWS). Heute muss sich die Union, müssen sich die sie betreibenden Nationalstaaten, immer noch höchst Bedacht auf ihrem politischen Primat gegenüber dem gemeinsam so Notwendigen, der unangenehmen, scheinbar unlösbaren Frage des Wie weiter? stellen. Eine andauernde Verzögerungstaktik ist im Gange, kann und wird aber keine Lösung bringen. Ist im Sinne Monnets Europa, die Union noch zu retten? Welche Folgen hätte der Rückschritt auf eine reine Wirtschaftsgemeinschaft? Dieser Beitrag setzt sich zum Ziel, einen alternativen Weg vorzuschlagen, der ganz im Sinne der richtigen Erkenntnis von Monnet bereits vor vielen Jahrzehnten unerlässlich schien: mehr Bundesstaat als Staatenbund (Abb. 1).

\section{Die Krise der Europäischen Union}

Es ist eher ungewöhnlich, dass sich zwei der führenden politikwissenschaftlichen Größen Deutschlands, Ulrich Menzel (2019) und Herfried Münkler (2019) mit profunden, wichtigen Wortmeldungen zur derzeitigen Krise der Weiterentwicklung Europas, der Europäischen Union an die Öffentlichkeit wenden - auf jenem Niveau, das diese ernsthafte Lage verdient, ja verlangt. Während die Welt gespannt das Geschehen um SARS-CoV-2 verfolgt, verschlechtert sich die ohnehin schon äußerst angespannte globale Lage immer weiter, im Schatten des erstgenannten Problems liegend, nicht kurz- aber langfristig mit wesentlich ernsteren Konsequenzen drohend und nur bruchstückhaft, wenn überhaupt, wahrgenommen. Die Geschichte der

\footnotetext{
${ }^{1}$ Der Europarat wurde 1949, die EGKS 1951 gegründet.
} 


\section{VÖLKERRECHT}

- Eine Bevölkerung (Staatsvolk),

- Ein geographisch abgrenzbarer Teil der Erdoberfläche (Staatsgebiet)

- Eine stabile Regierung, die effektive Gewalt ausübt (Staatsgewalt).

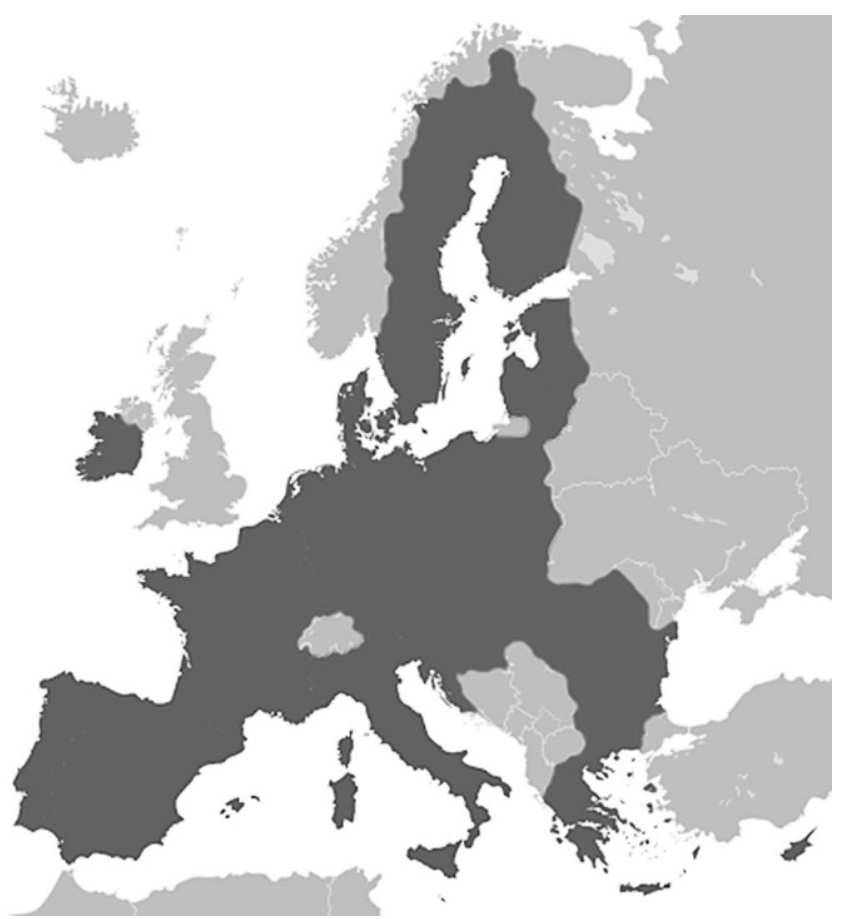

Abb. 1 Das Europa von Jean Monnet: Die Vereinigten Staaten von Europa auf Basis der EU-27. (Quelle: eigene Darstellung; Karte mod. nach Maix 2007)

sicherheitspolitischen Positionierung der europäischen Staaten (Gemeinschaft?) ist eine lange, unerfreuliche und immer dringlicher werdende. Schon die Gründerväter der Europäische Gemeinschaft für Kohle und Stahl (EGKS) haben anlässlich der Vision einer europäischen Einigung - nicht nur auf wirtschaftlicher, sondern konsequenter Weise auch auf der politischen und sicherheitspolitischen Ebene - das Wort geredet, dafür geworben, die Grundlagen geschaffen. Jean Monnet, der Franzose (!), war Zeit seines Lebens ein glühender Verfechter eines geeinten, politischen und zwischen den Blöcken - eigenständigen Europas. Alle diese Träume, Strukturen, Wünsche, ja Notwendigkeiten sind dem Zug der Zeit, der Schwäche der politischen Führungen in den Nationalstaaten und der Gleichgültigkeit der europäischen Völker zum Opfer gefallen und müssen heute, notwendiger denn je, als de facto undurchführbar beurteilt werden.

\section{Zwei Lösungsansätze nach Menzel und Münkler}

Menzel und Münkler verfolgen verschiedene Ansätze, haben unterschiedliche Empfehlungen für die Realisierung der europäischen Sicherheit bzw. der Sicherheit der EU in der neuen geopolitischen Lage. Sie sind sich - man kann durchaus meinen, es war nicht anders zu erwarten - in der grundsätzlichen Analyse und der historisch-ent- 
wicklungsmäßigen Ausgangslage und Entwicklung einig. Ebenso findet man kaum Trennendes hinsichtlich der Fortsetzung nach dem Zweiten Weltkrieg bis in den Zeitraum der Implosion der Sowjetunion, den des Endes des Kalten Krieges. Während dann Menzel für die nächsten zehn bis zwanzig Jahre fünf erwartbare Szenarien (mit einem Schwerpunkt im Einbezug der Rolle Chinas) analysiert und letzten Endes als wahrscheinlichstes sein Szenario fünf „Mit den USA nach Trump - der beste Fall unter vielen schlechten" (Menzel 2019) propagiert und als das glimpflichste bezeichnet, ändert Münkler schon rasch Analyse und dann natürlich Ansatzvorschläge in Hinblick auf eine euro-asiatische, kontinentalere Sicht unter dem Titel „Europas Wahl zwischen Wertebindung und Geopolitik“ (Münkler 2019). Er legt Wert auf die Feststellung, dass „keine europäische Geopolitik ohne strategisches Zentrum“ (Münkler 2019) möglich wäre und hier die Gegensätze zwischen Deutschland und Frankreich historisch und realpolitisch sowohl bei Beurteilung der Vergangenheit als auch der zukünftigen sicherheitspolitischen Herausforderungen sehr, es scheint unüberbrückbar unterschiedlich wären. Seiner Ansicht nach muss zwischen ,einem Primat der Wertebindung und einem Primat der geopolitischen Konstellation“ (Münkler 2019) entschieden werden. Und er macht auch kein Geheimnis daraus, dass hier Frankreich (seiner Ansicht nach besonders Präsident Emmanuel Macron) eine starke Einbindung Russlands im Sinne eines strategischen Partners „zu einem Bestandteil einer europäischen Sicherheitsordnung “ (Münkler 2019) anstrebt, da ein genereller Konsens innerhalb der Union und insbesondere der mit Deutschland als äußerst schwierig erreichbar zu beurteilen wäre.

\section{Die EU als Achsenmacht?}

The first lesson the student of international politics must learn and never forget is that the complexities of international affairs makes simple solutions and trustworthy prophecies impossible. (Morgenthau 1948, S. 6)

Das strategische Dreieck - ein Beurteilungs- und Analyseinstrument, ursprünglich aus der Wirtschaftstheorie stammend (Dittmer 1981) - ist von Yuh-Feng Lee (2003) mit dem Beispiel des Dreiecksverhältnisses USA, Volksrepublik China (VRC) und Republik China (Taiwan) in die Politikwissenschaft überführt worden. Es ist auch auf die vorliegende Situation/Analyse sehr gut anwendbar.

Der Dreieckstheorie nach kann die Achse durch geschicktes Variieren ihrer Positionen ein Gleichgewicht aufrechterhalten. Die Freundschaften der Achse mit den beiden (untereinander feindlichen) Flügeln (+) sind für den positiven Austausch (Werte/Güter) ebenso notwendig wie die negativen Beziehungen der Flügel (-) untereinander - sie halten die Flügel vom Abschluss einer gegen die Achse gerichteten Allianz ab, die das Dreieck massiv verändern würde. Die Achse wiederum kann mit den Beziehungen zu den Flügeln spielen, um zu versuchen, ihre eigene Position ständig zu verbessern. Sie muss aber ebenso die eigene Attraktivität für beide Flügel aufrechterhalten und dabei permanent die eigene militärische Überlegenheit im Auge haben. Dabei werden nun die Flügel- und Achsenpositionen verfeinert: Partner und Außenseiter treten auf den Plan. Soweit die Theorie dieses Modells. 


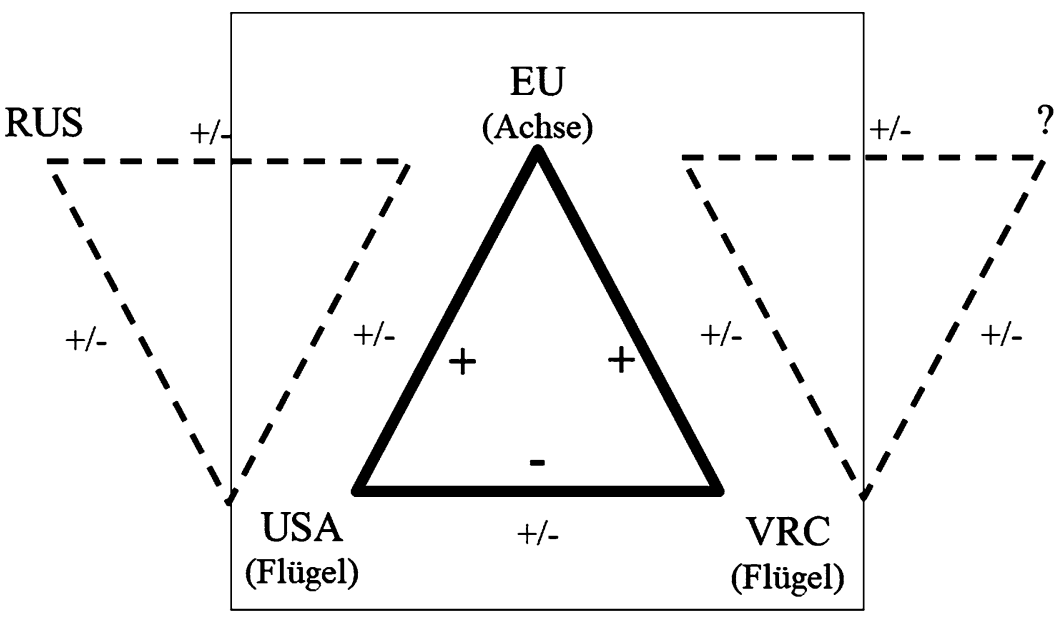

\section{Peripherie}

Kern

Peripherie

Abb. 2 Das Strategische Dreieck: Modell der Achsenmacht EU. (Quelle: eigene Darstellung nach Lee 2003)

Für die vorliegende Betrachtung muss - mit einem Modell der Achsenmacht EU (Abb. 2) - militärische Macht/hard power zum Zwecke der Spielbarkeit durch Wirtschafts- und Wertemacht/soft power ersetzt werden, wobei Wirtschaftsmacht durchaus hard- und soft-orientiert auftreten kann. In der Kernlage entspricht nun der grundsätzliche Ansatz den realen Möglichkeiten, erlauben doch gerade das politische und dabei insbesondere das sicherheitspolitische Defizit der EU keinen anderen (hard power) Ansatz. Ein Grundverständnis des Power-Konzeptes (hard, soft und letztlich smart power) nach Joseph Nye (2004) kann und wird hier vorausgesetzt. Das ursprünglich duale Verhältnis von Staatsmacht im Hard Power-Bereich, allgemein als militärische Macht gesehen, zu kulturell-philosophischer soft power und deren Interdependenz ist heute durch einen dritten Begriff ergänzt worden: smart power, also die Summe aus hard und soft power, richtig eingesetzt. Damit ist es aber noch nicht getan. Das unerlässliche Einbeziehen der Peripherie - mehr der Fall bei Menzel, der ja direkt die Präsenz und die Machtauseinandersetzung USA-China anspricht, aber natürlich auch bei Münkler keineswegs negiert oder minder bewertet, allerdings weniger im direkten Fokus stehend - zeigt nun klar die Vervielfältigung der Herangehensweisen, Möglichkeiten und Unwägbarkeiten auf. Münklers (2019) duale Grundoption ,mehr Westen oder mehr Osten wagen“ oder Menzels fünfte Option, die seiner Ansicht nach realistischste, nämlich durch eine gestärkte Partnerschaft mit den USA den übermächtig-drohenden chinesischen globalen Einfluss einzudämmen - beide Möglichkeiten, und da herrscht hohe Übereinstimmung, lassen sich mit den derzeitigen politisch-sicherheitspolitischen (bedeutet militärischen) Schwächen der Union keinesfalls realisieren. Achsenpositionen im strategischen Dreieck lassen sich nicht ausschließlich als soft power definieren, geschweige denn realpolitisch verwerten. Münklers Forderung - ob mehr Westen oder Osten - nach einem strategischen Zentrum verlangt zwingend politische Einigkeit über ein dann 
zu schaffendes europäisches, nicht nationales sicherheitspolitisch-militärisches Potenzial entsprechender Größe und Stärke, mit hoher Einsatzbereitschaft. Ohne diese Voraussetzungen bleibt der Wunsch, alleine auf soft power aufbauend ein global player zu sein, ein unerfüllbarer Traum.

Auf welchen Grundlagen kann nun versucht werden, eine dritte Option anzustreben? Es sind primär drei Ebenen, deren interaktives Zusammenwirken hier betrachtet und untersucht werden muss: Politik, Sicherheitslage und die wirtschaftliche Basis. Zuvor ist jedoch ein kurzer Rückblick auf den nun über 70 Jahre andauernden Weg zu mehr europäischer Einigung im Korsett einer Union - mit welchem Ziel? unerlässlich.

Europarat (1949), EGKS (1951), die Römischen Verträge (Gründung EWG und EURATOM, 1958/59), die Verträge von Maastricht (1992/93) und Amsterdam (1997/99), die Grundrechtecharta (2000), der Vertrag von Nizza (2001/03), das Scheitern der europäischen Verfassung (2005), der Vertrag von Lissabon (2007/09) und der definitive Austritt des Vereinigten Königreichs (2016/20) sind Etappen von großer Bedeutung, geprägt von einem grundsätzlichen Wollen der Europäer, allerdings nicht umfassend in Hinblick auf gemeinsames Handeln, sondern vielmehr unter maximalem Erhalt nationalstaatlicher Macht unter Akzeptanz wirtschaftlichsozialer Verknüpfung, keinesfalls politisch-gemeinschaftlicher. Der amerikanische Kollege Bruce Jones ${ }^{2}$ (2020, S. 5) hat in einem Essay über die Auseinandersetzung um die globale Führungsposition zwischen den Vereinigten Staaten und China in einem bemerkenswerten Satz die EU-Fähigkeiten auf der geopolitisch-strategischen Weltbühne wie folgt definiert:

Europe, [nicht die EU?!, Anmerkung des Autors] for its part, is meeting the rise of China, the aggression of Russia, and the unilateralism of the Unites States with a combination of bewilderment, nostalgia and hesitant exploration of selfhelp approaches that so far fall well short of a credible strategic response.

Dieser eine Satz fasst 70 Jahre erfolgloser Bemühungen um politische Einheit, vor allem nach außen, zusammen. Unsere Analyse muss nun jene Bereiche/Probleme erfassen, die als Grundlage der sicherheitspolitischen Lage Europas/der Union von Bedeutung sind.

Abbildung 3 verdeutlicht klar die Notwendigkeit zu einer radikalen Kursänderung; Jean Monnet hat von Beginn an die Meinung vertreten und gefordert, dass eine Weiterentwicklung der zunächst klug als Eintrittsszenario gewählten wirtschaftlichen Grundlage zu politischer Einigkeit und koordiniertem Auftreten nach außen stattfinden muss. Er legte mit der Westeuropäischen Union (WEU) auch einen formellen Rahmen und vertrat offen das Konzept der zu errichtenden Vereinigten Staaten von Europa (VSE). Das von ihm im Oktober 1955 gegründete Aktionskomitee für die Vereinigten Staaten von Europa (ACUSE) hatte die Billigung zahlreicher

\footnotetext{
2 Bruce Jones ist Director und Senior Fellow, International Order and Strategy of the Foreign Policy Program, Brookings Institution (Washington, DC.).
} 


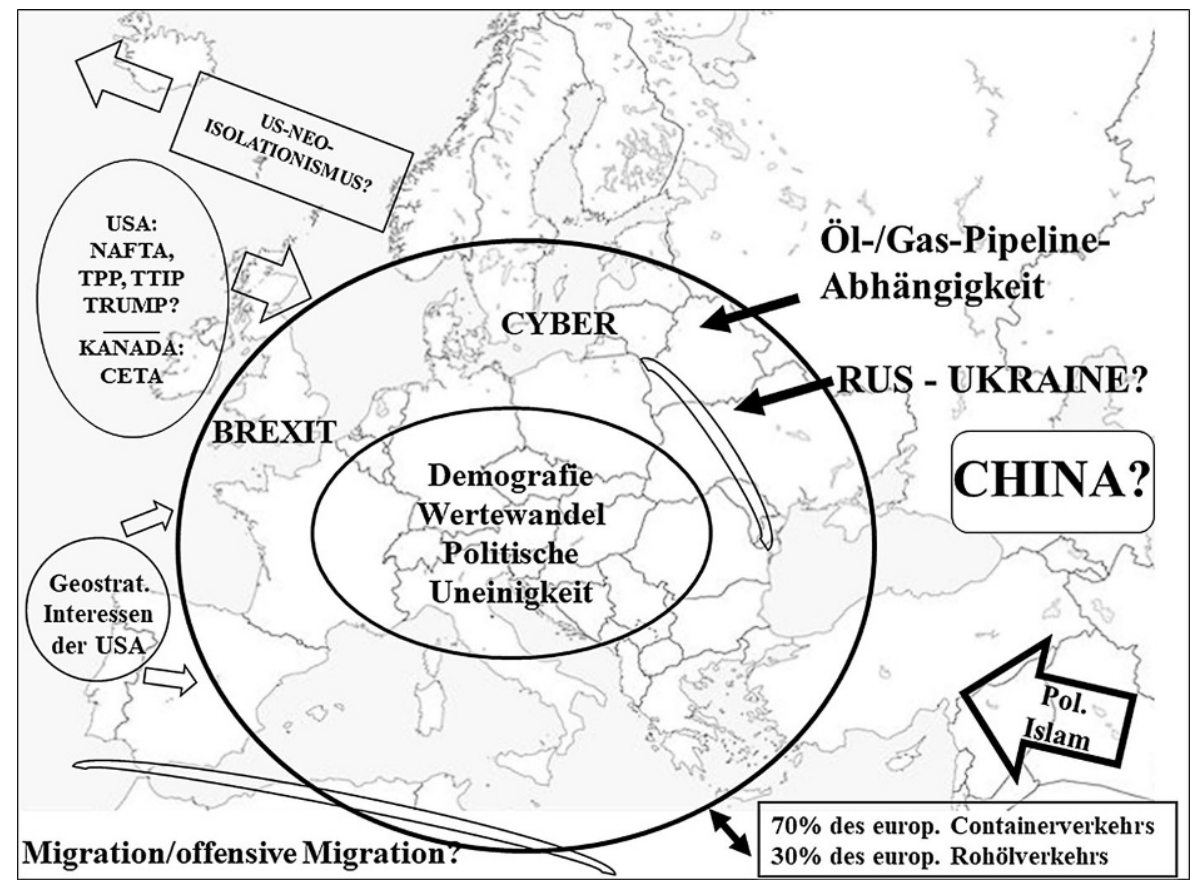

Abb. 3 Konfliktlage der Europäischen Union. (Quelle: eigene Darstellung nach Peischel, persönliche Kommunikation)

europäischer Politiker ${ }^{3}$ (z. B. Amintore Fanfani, Kurt Georg Kiesinger, Guy Mollet, René Pleven, Herbert Wehner später Willy Brandt, Edward Heath, Leo Tindemanns), konnte sich aber in den 20 Jahren des Bestehens nicht wirklich gegen die nationalen Strömungen in den Institutionen und den Völkern der späteren Union durchsetzen. Monnet selbst vollzog im Mai 1975 die Auflösung unter der Feststellung, dass der damals auch unter seiner Mithilfe geschaffene Europäische Rat (die Staats- und Regierungschefs) das richtige Gremium für die weitere Arbeit in Richtung Einheit und durch keine andere Institution ersetzbar wäre. Aus den damals neun Mitgliedern sind heute 27 geworden - und die als Bindeglied gedachte Einstimmigkeit hat sich seither als eines der größten Hindernisse für die Weiterentwicklung der politischen Einigkeit erwiesen. Das liegt auch und hauptsächlich daran, dass es fortan an visionären Staatspolitikern in den führenden Mitgliedsstaaten mangelte und zu raschen Erweiterungen ohne entsprechende Strukturen neuer Mitgliedsstaaten gekommen ist.

\footnotetext{
${ }^{3}$ Im vorliegenden Artikel wird, abweichend vom ZfAS-Standard, bei personenbezogenen Substantiven die männliche grammatikalische Form verwendet. Der Autor schließt damit Personen jeden Geschlechts gleichermaßen ein.
} 
Abb. 4 Grand Strategy eines vereinigten Europas. (Quelle: eigene Darstellung)

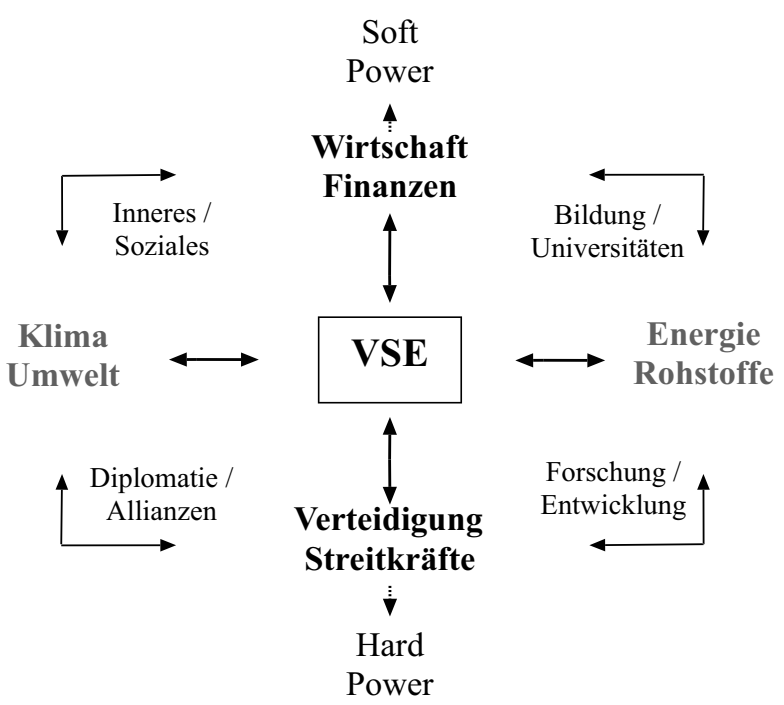

\section{Voraussetzungen für einen gemeinsamen Weg}

Bei aller berechtigten Kritik soll und darf nicht außer Acht gelassen werden, dass diese Union in den Jahren ihres Bestehens einer, vielleicht gar der Hauptaufgabe aus ihren Gründungszielen, nämlich neuerliche kriegerische Auseinandersetzungen zwischen den europäischen Staaten zu verhindern, voll entsprochen hat. Das ist und darf allerdings nicht als bequeme Selbstverständlichkeit immerwährend vorausgesetzt werden. Wie sollte die EU also nun vorgehen, welche Schritte setzen, welche Ziele vorgeben? Wie kann verhindert werden, dass sich die weitere Marginalisierung der Union auf der globalen Bühne fortsetzt, die sicherheitspolitisch überlebensnotwendige Allianz mit den Vereinigten Staaten immer mehr Risse zeigt, die Union nicht in der Lage ist, das kleinste sicherheitspolitische Problem - Abbildung 4 zeigt die entsprechenden Felder im Sinne einer Grand Strategy klar und deutlich auf alleine zu lösen und statt einem Vasallen endlich und im eigenen Interesse zu einem Partner im transatlantischen Bündnis zu werden? Der vorliegende Beitrag hat Politik, Sicherheitslage und wirtschaftliche Basis als Eckpfeiler zur Lösung identifiziert. Ein seriöses Projekt (im Sinne Monnets) der Vereinigten Staaten von Europa bedarf vieler Voraussetzungen, um zu gelingen. Nun ist aber - wieder genügt ein Blick auf Abbildung 4 - klar, dass die heutigen Voraussetzungen sich durch einen einzigen zusätzlich-erschwerenden Umstand von denen zum Zeitpunkt der Beendigung des ACUSE (1975!) unterscheiden: den Faktor Zeit. Es bleibt kaum mehr Zeit, um eine derart anspruchsvolle, herausfordernde Änderung des gemeinsamen Weges als Union zu planen, zu erklären und rasch umzusetzen. Das gute alte Argument der letzten Jahrzehnte bei derartigen Gesprächen und Diskussionen, dass man eben mehr Zeit bräuchte, ist nicht mehr anwendbar. Es ist für die Union nicht fünf vor, sondern bereits 15 nach 12 Uhr mittags 
Ein derartig epochales Vorhaben bedingt vor allem an der Spitze der führenden Staaten der Union - primär Deutschland und Frankreich, aber auch als aktive Mitspieler Benelux, Italien und die nordischen Staaten, vielleicht auch Österreich - Staatspersönlichkeiten, die in der Lage sein müssen, ihre Nationen von der unabänderlichen Notwendigkeit dieses Schritts zu überzeugen und ihr persönliches, politisches Schicksal daran festmachen. Es hat in der Vergangenheit solche Politiker gegeben - in vielen europäischen Nationen. Die heutige Konstellation zeigt eine vorsichtig-mögliche Option für Frankreich, allerdings eine eher ungünstige in Deutschland. Nur die gleiche, abgestimmte Vorgangsweise zweier Staatspersönlichkeiten gleichzeitig könnte hier etwas bewirken; dann würden sich sehr wahrscheinlich, wie schon erwähnt Benelux, Italien und Spanien vielleicht nicht sofort aber grundsätzlich schon, die nordischen Staaten sowie aus klar und primär sicherheitspolitischen Gründen Polen, vielleicht auch Österreich, anschließen und den Kern der VSE bilden.

Schon Monnet hat sich dieser Grundidee tief verpflichtet gefühlt; nach ihm, nach dem Ende der ACUSE, hat es außer dem belgischen Liberalen Guy Verhofstadt kein Politiker mehr gewagt, dieses Thema konsequent zu verfolgen oder gar zu propagieren: Ab 1975 und erst viel später, nach dem Scheitern des Verfassungsansatzes 2005 (Frankreich und die Niederlande lehnten ab), hat Verhofstadt 2006 sein Buch Die Vereinigten Staaten von Europa vorgelegt und für die so bitter notwendige politische Einheit geworben - vergebens, wie Monnet. Kann ein neuer Ansatz erfolgreich sein? Wie müsste vorgegangen werden? Die Verfassungsfrage dieses Bundesstaats müsste - conditio sine qua non - in einem Erstschritt von eben Deutschland und Frankreich als Vorleistung ausgearbeitet, ein Entwurf den beiden Nationen erläutert und am gleichen Tag X zur Direktwahl beiden Nationen vorgelegt werden.

\section{Eine neue staatspolitische Struktur}

Das in Abbildung 5 dargestellte Konstrukt erfüllt alle Voraussetzungen eines auf Subsidiarität und starker Außenwirkung mit einheitlicher Stimme auftretenden players und kann - alles ist verhandelbar - zunächst sogar Teil der EU sein/bleiben. Ziel wäre es, in Schritten beitrittswilligen EU-Staaten nach einfacher Abstimmung im Beitrittsstaat (unter Preisgabe der Nationalität, nicht des Staatsgebietes oder Volkes) nach dem amerikanischen Modell federal states ein weiterer MitgliedsStaat des Staatenbundes zu werden. Auch die leidige Pass- und Nationalsprachenfrage ist bei gutem Willen leicht lösbar. Die VSE ist grundsätzlich zweisprachig Englisch, de facto Weltsprache, als federal language und im jeweiligen Bundesstaat dessen nationale Sprache - die Schweiz hat vier nationale Sprachen (!). Dazu kämen ein europäischer Pass, europäische Staatsbürgerschaft und im Bereich federal die klassischen Elemente: Äußeres, Verteidigung, föderale Polizeikräfte, Justiz. Alles andere Administrative regeln die (National-)Staaten. Warum sollten die Menschen - zunächst in Deutschland und Frankreich - diesem Vorschlag zustimmen? Weil ihnen ihre jeweiligen Regierungen erklären müssten, wie und ob sich ihre kleinen Nationalstaaten, zwar Mitglieder einer lockeren Handelsorganisation EU (ohne jede starke politische Macht, viel soft- aber keine wirkliche hard-power), in einer Welt 


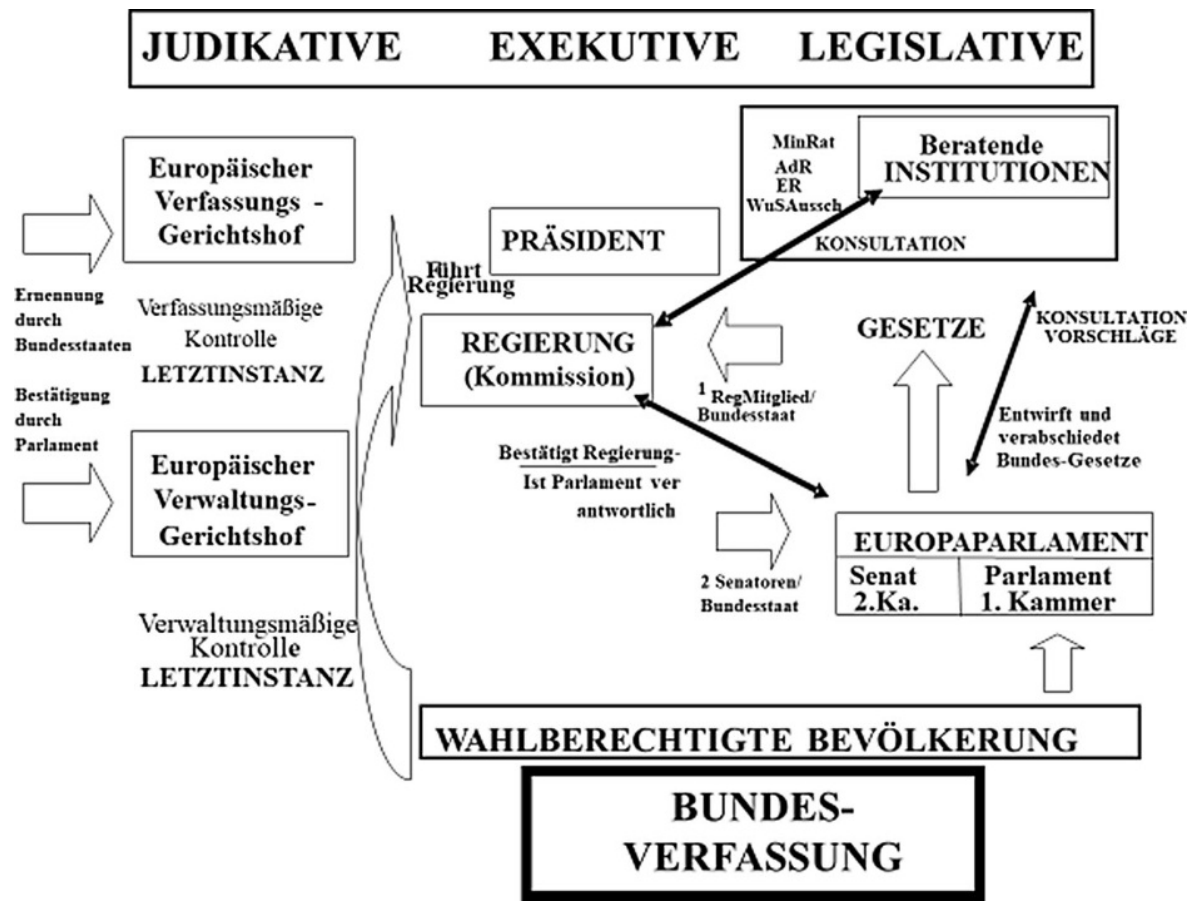

Abb. 5 Politisches Modell der Vereinigten Staaten von Europa. (Quelle: eigene Darstellung)

der Zukunft einfach nicht mehr als Exportweltmeister oder grande nation behaupten werden können.

\section{Umsetzung und Herausforderungen}

Die hier vorgestellte dritte Option erscheint illusorisch, als nicht realisierbar, unmöglich. Sie ist aber aus den vielen genannten Gründen ohne Alternative - es sei denn, die europäischen Nationalstaaten geben sich mit kleinen, unbedeutenden Nebenrollen auf der Weltbühne zufrieden. Andere werden dann die Regeln und Normen allein bestimmen, nach denen die Welt der Zukunft, der nahen, funktionieren wird. Dies ist umso bedeutender, als zuletzt ein zusätzlicher, globaler Faktor erwähnt werden muss, der in Bezug auf das Gewicht der Union in den kommenden Jahrzehnten von ganz außerordentlicher Bedeutung sein wird: Haltung, Gewicht und Bedeutung bei den unerlässlichen Maßnahmen der Menschheit in Bezug auf ihre Umwelt. Dieses komplexe Thema, das natürlich über die Lösungsfähigkeit durch einen oder einige Nationalstaaten, gleich welcher Größe, politischer, militärischer oder jedweder anderen Bedeutung weit hinausgeht, kann nicht mehr ohne gemeinsame, kooperative Zugänge der global player gelöst werden. Und bei diesen nicht dabei zu sein, hieße, dass wichtige Entscheidungen und Maßnahmen ohne entsprechende oder mit geringer Berücksichtigung der Europäer gefällt und umgesetzt würden. Das muss 
die Politik der Nationalstaaten und der EU den Europäern erklären - schonungslos und ehrlich. Die Schwierigkeiten dieses Motivationsvorhabens sind enorm. Es geht dabei vorrangig um das Vermitteln des notwendigen, vernetzten Ansatzes über alle Bereiche des (nationalen) staatlichen Handelns, ohne den die Umweltproblematik nicht gelöst werden kann. Das Schlüsselargument dabei ist, dass - unabhängig ob ein Staat/Staatenbund zustimmt oder nicht - ein gemeinsames Handeln unerlässlich ist. Dies überfordert klarer Weise viele Menschen und führt zu Ablehnung, Gleichgültigkeit, Verdrängen und genau dann ist eben der Staatspolitiker gefordert.

\section{Fazit}

Vor allem auf Grund der in Kapitel 7 genannten Herausforderungen benötigen die Europäer eine Struktur des gemeinsamen Handelns in den staatlichen Kernbereichen, keine EWG mit impotenten Kommissionen, Räten etc. Die Entscheidungsund Handlungsfähigkeit sind das Kernelement eines global players. Ein dritter Weg scheint die letzte Chance zu sein, rechtzeitig diese Struktur einzuführen. Da festgestellt wurde, dass de facto kein Weg an dieser Einigung vorbeiführt, will Europa nicht in zwei oder drei Dekaden eine unbedeutende Ansammlung mittlerer und kleiner Nationalstaaten sein, ohne Gewicht und Macht - jawohl, Bedeutung auf der Weltbühne wird nicht nur in soft- sondern auch in hard power gemessen -, dann muss mit der Umsetzung begonnen werden. Viele Fragen stehen offen. Kann Macron Frankreich in einer klugen Balance zwischen grande nation-Denken und der doch eher restriktiven Realität der französischen Wirtschaft mit einem zukünftigen deutschen Staatspolitiker diesen Prozess irreversibel aufstellen? Folgen andere Staaten diesem Weg? Ist die zukünftige amerikanische Präsidentschaft bereit, die Union als echten Partner zu akzeptieren? Geben uns andere global player mit eigenen, natürlich nicht immer entgegenkommenden Interessen genug Zeit, um unser System in Ordnung zu bringen?

Es gibt viele Fragen und Visionen, kaum konkrete Antworten ${ }^{4}$. Das berühmte Zitat von Nelson Mandela „Die Dinge scheinen immer unmöglich, bis sie getan sind" müsste Kompass und Motivation sein, den hier beschriebenen Schritt eines alternativen Weges zu gehen.

\section{Literatur}

Dittmer, L. (1981). The strategic triangle: an elementary game-theoretical analysis. World Politics, 33(4), $485-515$.

Jones, B. (2020). China and the return of great power strategic competition. Washington, DC: Brookings. Lee, Y.-F. (2003). Die Taiwan-Frage im Kontext der US-Strategie für Ostasien-Pazifik nach dem Ende des Ost-West-Konflikts (1990-2000). Dissertation, Berlin: Humboldt-Universität zu Berlin.

Maix (2007). A political Map of Europe in SVG format. Wikimedia Commons. CC-BY-SA-3.0. https:// commons.wikimedia.org/wiki/File:Blank_map_of_Europe_cropped.svg. Zugegriffen: 7. Okt. 2020.

\footnotetext{
${ }^{4}$ Es sei hier die Anekdote erwähnt, nach der der ehemalige österreichische Bundeskanzler Franz Vranitzky auf die Frage nach Visionen in der Politik geantwortet haben soll: „Wenn Sie Visionen haben, gehen Sie zum Arzt".
} 
Menzel, U. (2019). Welt im Übergang, Europa in der Krise. Vom amerikanischen zum chinesischen Jahrhundert. Blätter für deutsche und internationale Politik. https://www.blaetter.de/ausgabe/2019/ september/welt-im-uebergang-europa-in-der-krise. Zugegriffen: 7. Okt. 2020.

Morgenthau, H. (1948). Politics among nations: the struggle for power and peace. New York: Alfred Knopf.

Münkler, H. (2019). Mehr Westen oder mehr Osten wagen? Europas Wahl zwischen Wertebindung und Geopolitik. Blätter für deutsche und internationale Politik. https://www.blaetter.de/ausgabe/2019/ oktober/mehr-westen-oder-mehr-osten-wagen. Zugegriffen: 7. Okt. 2020.

Nye, J.S. (2004). Soft power: the means to success in world politics. New York: Public Affairs.

Verhofstadt, G. (2006). Die Vereinigten Staaten von Europa. Manifest für ein neues Europa. Eupen: GEV. 